\title{
Skin type analysis based on the new questionnaire in Korean women
}

\author{
Byeong Jin Park, Young Wook Ko, Dong Uk Cheon, Su Jin Oh, \\ JooYeon Ko, Young Suck Ro, Jeong Eun Kim
}

\section{Department of Dermatology, Hanyang University College of Medicine, Seoul, Korea}

Over the past decades, several terms, including dry, oily, combination and sensitive, have historically been used to characterize skin types. However, these are not based on the evident dermatologic definition, which usually includes subjective and psychological properties. While cosmetic laser procedures are commonly performed in Korean women, the safety profile results frequently differ compared with Western patients. The aim of this study was to establish a new practical questionnaire to classify skin subtypes in Korean women to establish treatment and skin care guidance.

Eight experts from seven university hospitals developed consensus statements about skin types and created a questionnaire that consisted of 11 items (Figure 1). The content was categorized into four major subtypes: dry or non-dry; oily or non-oily; sensitive or non-sensitive; pigmented or non-pigmented. A total of 512 patients aged 20 to 65 years were included and completed the questionnaire. Correlations with age, skin Fitzpatrick's phototypes, and dermatologic comorbidities on the face were analyzed using Chi-square test and Chi-square trend test.

141 of 512 patients (27.5\%) were in the third decades of age, the most common group, followed by fourth (27.1\%), fifth $(21.3 \%)$, sixth (15.4\%), seventh (2.1\%) decade groups and the patients' mean age was 37.4 years. Korean women commonly have oily skin but complain of dryness after cleansing. They are especially sensitive to skin care products and prone to developing pigmentary lesions. Table 1 shows the correlations between age, modified Fitzpatrick's skin phototype and dermatologic comorbidities.

Table 1. Associations with age, dermatologic comorbidities, and skin phototype

\begin{tabular}{|c|c|c|c|c|c|c|c|c|c|c|c|}
\hline \multirow{2}{*}{${ }^{*} p<0.05$} & \multicolumn{2}{|c|}{ Dry skin } & \multicolumn{2}{|c|}{ Oily skin } & \multicolumn{5}{|c|}{ Sensitive skin } & \multicolumn{2}{|c|}{ Pigmented skin } \\
\hline & Q1 & Q2 & Q3 & Q4 & Q5 & Q6 & Q7 & Q8 & Q9 & Q10 & Q11 \\
\hline Age & 0.906 & $0.012^{*}$ & $<0.001^{\star}$ & $0.012^{*}$ & 0.188 & $0.002^{\star}$ & 0.052 & $0.007^{\star}$ & & $0.004^{\star}$ & 0.238 \\
\hline $\begin{array}{l}\text { Dermatologic } \\
\text { Cormorbities }\end{array}$ & 0.791 & 0.774 & 0.642 & 0.079 & $<0.001^{\star}$ & $0.042^{*}$ & $<0.001^{*}$ & $<0.001^{*}$ & & 0.599 & 0.490 \\
\hline phototype & 0.946 & 0.063 & 0.289 & 0.264 & 0.631 & 0.798 & 0.435 & 0.899 & & $0.002^{*}$ & $0.001^{*}$ \\
\hline
\end{tabular}

